\title{
PLANNING EMPTY CONTAINER RELOCATIONS UNDER UNCERTAINTY
}

\author{
W.L. Bean ${ }^{1}$ and J.W. Joubert ${ }^{1,2}$ \\ ${ }^{1}$ Logistics and Quantitative Methods \\ CSIR Built Environment, South Africa \\ wbean@csir.co.za \\ ${ }^{2}$ Department of Industrial and Systems Engineering \\ University of Pretoria, South Africa \\ johan.joubert@up.ac.za
}

\begin{abstract}
Reusable packaging often accumulates downstream in supply chains, resulting in nonrevenue-earning relocations. This paper presents a reliable optimisation model under supply and demand uncertainty. Instead of using deterministic forecasted values, we propose a fixed recourse stochastic program that embeds the approximated uncertainties within the decision model. Results do indeed prove to be more reliable, and thus useful to industry. We also investigate the model's sensitivity to the difference in weighting of inventory versus stock-out penalties. The valuable role of Operations Research in industry and of optimisation, specifically - is affirmed. Though uncertainties cannot be eradicated, planned relocations can be done more reliably.
\end{abstract}

\section{OPSOMMING}

Herbruikbare verpakking bou gereeld aan die onderpunt van voerkanaalnetwerke op met nie-verdienste-genererende herskuiwings tot gevolg. Hierdie artikel bied 'n betroubare optimeringsmodel aan wat vraag- en aanbodonsekerheid aanspreek. In plaas van deterministiese vooruitskattings, stel ons 'n vaste verhaling stogastiese program voor waar die benaderde onsekerheid in die model self vasgevang word. Resultate is inderdaad meer betroubaar en dus bruikbaar vir industrie. Ons beskou ook die sensitiwiteit van voorraadteenoor vooraadlose strafgewigte. Die waardevolle rol van Operasionele Navorsing - en meer spesifiek, optimering - word bevestig. Alhoewel onsekerheid nie uitgewis kan word nie, kan meer betroubare herskuiwings beplan word. 


\section{INTRODUCTION}

Although the term logistics is widely used and recognised, Coyle et al. [6] provide four useful subdivisions: business logistics, military logistics, event logistics, and service logistics. The focus of this paper, business logistics, is a process in the supply chain that plans, manages, and implements the effective and efficient storage and flow of goods, services, and information from point of origin to the point of consumption in order to satisfy customer requirements.

The product concerned in this paper, however, is not a consumable or service. Rather, this paper focuses on the reusable packaging used to store, handle, protect, and transport goods through the supply chain. Examples of such reusable packaging include, but are not limited to, pallets, containers, trolleys, bins, and custom made crates. Once products arrive in their packaging at their destination, the reusable packaging tends to build up since there is not sufficient demand for products in the opposite direction. This requires reusable packaging to be relocated back upstream in the supply chain to where products are produced and packaged. Whereas products earn revenue, reusable packaging only incurs costs, requiring proper management to ensure cost minimisation $[9,10,11]$. Olivo et al. [14] cite an Italian survey estimating that the handling of empty shipping containers alone will increase from approximately US\$ 25 billion in 1999 to US\$ 50 billion in 2010. Proper management of reusable packaging can therefore provide cost-saving opportunities, providing scope for analytical decision support. For the remainder of the paper we will refer to all forms of reusable packaging types collectively as containers.

Whereas strategic decision models, such as the work by Crainic and Delorme [8], consider long-term decisions such as where depots should be located, this paper deals with the more operational decisions regarding the relocation of containers.

Operations Research (OR) literature abounds with models assisting companies with the management of forward product movement, but few models are concerned with the return leg of empty container relocations. One of the first contributions was the balanced transportation approach by Misra [13], considering a single commodity, rail cars. In the mathematical model the objective is to minimise the total cost of relocating empty containers. The objective is constrained by the available supply of and demand for empty containers at every station. Demand at every station must be met, while the total supply must equal the total demand, resulting in a balanced system.

One of the early Empty Container Allocation Problem (ECAP) contributions was by Crainic et al. [7], formulating models for deterministic single and multi-commodity problems, and providing a general modelling framework. Crainic and Delorme [8] use the formulation and provide a heuristic solution approach, while Choong et al. [5] later extend the formulation to include storage capacities and multiple capacitated transportation modes.

A multi-commodity network flow optimisation model was formulated by Holmberg et al. [12]. The optimisation model addresses capacity constraints and strictly adheres to arrival and departure times. Supply and demand of empty cars are assumed to be given, albeit forecasted. Another deterministic contribution, by Olivo et al. [14], formulates the dynamic model with hourly time-steps. The term dynamic in that contribution refers to a rolling horizon procedure used to address the uncertainty of supply, demand, transit times, and equipment failures. These parameters are, however, used as deterministic parameters. Empty containers are assumed to be delivered to the customer during the time-period in which they are required.

Birge and Ho [2] present a formulation of a stochastic, dynamic network problem with dynamic decisions, uncertain input flows, and congestion. Their contribution also considers a multi-period decision model where uncertainties are resolved period-by-period as time progresses. The model is applicable to situations where flows must be directed between nodes, and complete information about future demand is not known. Cheung and Powell [4] 
also use stochastic programming, developing an algorithm for multi-stage dynamic networks with random arc capacities. They use arcs to characterise the movement of vehicles between cities.

A two-stage stochastic approach was followed by Cheung and Chen [3] in their formulation of the ECAP. In the first stage they use demand, capacity, and supply as deterministic values, while they are treated as random variables in the second stage. The more complex the formulation of the problem, the more difficult it is to find exact and optimal solutions, especially for large problem instances (Rajgopal [15]).

In this paper we considered a network $\mathbf{G}=(\mathbf{D}, \mathbf{A})$, consisting of depot nodes $\mathbf{D}=\{1, \ldots, D\}$, and directed arcs $\mathbf{A}=\{1, \ldots, A\}$. A set $\mathbf{E}=\{1, \ldots, E\}$ of container types is to be relocated between the nodes using trucks of different types, denoted by set $\mathbf{L}=\{1, \ldots, L\}$, that are available on demand, each type $l \in \mathbf{L}$ with a different capacity and cost associated with it. Both supply and demand are assumed to be uncertain, yet follow known probabilistic distributions. We used a fixed recourse approach to address the uncertain demand, since we want to track the number of containers either in storage or in short supply, so as to incur appropriate recourse penalties.

We were able to solve a realistically sized instance of the problem exactly, and also tested the reliability and usability of the model through scenario analysis. Our contribution is significant in that it not only provides a quantitative measure of the realistic usefulness of the model, but also investigates and reports on the effect that inventory and shortage costs have on the reliability of the optimisation model.

The remainder of the paper starts with the stochastic formulation of the ECAP in Section 2, along with the conversion of the model into its deterministic equivalent. A numerical example is solved in Section 3, with a discussion on the quantitative measure of the model's reliability in Section 4. The paper concludes in Section 5 and provides a brief agenda for future research.

\section{STOCHASTIC MODEL FORMULATION}

Recall from the introduction that the model formulated is concerned with finding a minimum cost relocation plan of all $e \in \mathbf{E}$ containers between the supply and demand depots, $s, d \in \mathbf{D}$. It is important to note that supply and demand depots can, but will not necessarily, be the same depot. Although both the supply and demand at each of the depots are only known as probabilistic random variables, the relocation decisions are nonadaptive, implying that the relocation decisions are made before the realisation of the random demand, and the original decisions are not adjusted once the actual demand is realised. Relocations are done through assigning the containers to different truck types $l \in \mathbf{L}$, each type $l$ with a different cost and capacity. Since different trucks have different capacities, and the volume of each individual container in its collapsed form (if it is collapsible) is different, the number of containers transported per truck may vary. Consequently, the transportation cost for every container can not be specified, and the total cost is expressed in terms of the number of each truck type $l$ used for relocations. Trucks are rented from service providers on demand, and incur a fixed cost based on their type, causing less-than-truckload movements to incur the same cost as full loads.

To formulate the model, decision variables are defined as follows:

$x_{\text {sde }} \quad$ the number of type $e$ containers shipped from supply depot $s$ to demand depot $d$, where $s, d \in \mathbf{D}$ and $e \in \mathbf{E}$. 
$y_{\text {sdel }} \quad$ the number of type $e$ containers shipped from supply depot $s$ to demand depot $d$ using a truck of type $l$, where $s, d \in \mathbf{D}, e \in \mathbf{E}$, and $l \in \mathbf{L}$.

$n_{s d l} \quad$ the number of type $l$ trucks required from supply depot $s$ to demand depot $d$, where $s, d \in \mathbf{D}$, and $l \in \mathbf{L}$.

A number of relocation related parameter definitions are required. We let:

$c_{s d l}$ the given cost to transport a load of containers from supply depot $s$ to demand depot $d$ using a truck of type $l$, where $s, d \in \mathbf{D}$, and $l \in \mathbf{L}$.

$I_{d e} \quad$ the given opening inventory of container type $e$ at depot $d$, where $d \in \mathbf{D}$ and $e \in \mathbf{E}$.

$V_{d}^{D} \quad$ the given volumetric capacity (in $m^{3}$ ) of depot $d \in \mathbf{D}$.

$V_{l}^{T} \quad$ the given volumetric capacity (in $m^{3}$ ) of a truck of type $l \in \mathbf{L}$.

$W_{l}^{T} \quad$ the given weight capacity (in $\mathrm{kg}$ ) of a truck of type $l \in \mathbf{L}$.

$v_{e}^{C} \quad$ the given unit volume (in $\mathrm{m}^{3}$ ) of a collapsed container of type $e \in \mathbf{E}$.

$w_{e}^{C} \quad$ the given unit weight (in $\mathrm{kg}$ ) of a container of type $e \in \mathbf{E}$.

Related to the uncertain supply and demand, we let:

$\tilde{\zeta}_{d e}$ the given random vector describing the supply of type $e$ containers at depot $d$, where $d \in \mathbf{D}$ and $e \in \mathbf{E}$.

$\tilde{\eta}_{d e} \quad$ the given random vector describing the demand of type $e$ containers at depot $d$, where $d \in \mathbf{D}$ and $e \in \mathbf{E}$.

$u_{d e}\left(\widetilde{\zeta}_{d e}, \widetilde{\eta}_{d e}\right)$ the number of type $e$ containers in inventory at depot $d$ at the end of the period, expressed as a function of both the random supply and demand of type $e$ containers at depot $d$, where $d \in \mathbf{D}$ and $e \in \mathbf{E}$.

$h_{d e}\left(\widetilde{\zeta}_{d e}, \widetilde{\eta}_{d e}\right)$ the number of type $e$ containers short at depot $d$ at the end of the period, expressed as a function of both the random supply and demand of type $e$ containers at depot $d$, where $d \in \mathbf{D}$ and $e \in \mathbf{E}$.

$o_{d e} \quad$ the given unit holding cost for type $e$ containers at depot $d$, where $d \in \mathbf{D}$ and $e \in \mathbf{E}$.

$r_{d e} \quad$ the given unit shortage penalty for type $e$ containers at depot $d$, where $d \in \mathbf{D}$ and $e \in \mathbf{E}$.

The objective of the model is to minimise the total cost of relocating empty containers. Also included are the expected inventory and shortage costs - that is, the recourse as a result of the uncertain supply and demand. Formulated in (1) is the objective function expressed as a stochastic program with recourse:

$$
\min z=\sum_{s, d \in \mathbf{D}} \sum_{l \in \mathbf{L}} c_{s d l} n_{s d l}+\sum_{d \in \mathbf{D} e \in \mathbf{E}} \sum_{\left(\widetilde{\zeta}_{d e}, \tilde{\eta}_{d e}\right)}\left[o_{d e} u_{d e}\left(\widetilde{\zeta}_{d e}, \widetilde{\eta}_{d e}\right)+r_{d e} h_{d e}\left(\widetilde{\zeta}_{d e}, \widetilde{\eta}_{d e}\right)\right]
$$


where $E_{\left(\widetilde{\zeta}_{d e}, \widetilde{\eta}_{d e}\right)}$ represents the expected value with respect to the realisations of the random supply, $\widetilde{\zeta}_{d e}$, and demand, $\widetilde{\eta}_{d e}$, for type $e$ containers at depot $d$. We revert to a two-stage fixed recourse approach to address the stochastic demands, and the resulting problem is then an ordinary deterministic Mixed Integer Program (MIP). The $\alpha^{\text {th }}$ realisation of the random supply is denoted by $\zeta_{d e}^{\alpha}$, which has a finite discrete distribution $\left\{\left(\zeta_{d e}^{\alpha}, p_{d e}^{\alpha}\right), \alpha=1, \ldots, R\right\}$ with $p_{d e}^{\alpha}>0 \forall \alpha$, and where $R$ is a given number of equidistant partitions with which the random distribution is approximated. Similarly the $\beta^{\text {th }}$ realisation of the random demand is denoted by $\eta_{d e}^{\beta}$, which has a finite discrete distribution $\left\{\left(\eta_{d e}^{\beta}, p_{d e}^{\beta}\right), \beta=1, \ldots, R\right\}$ with $p_{d e}^{\beta}>0 \forall \beta$.

With this approximation, we can alter the definition of the inventory and shortage variables, so we let:

$u_{d e}^{\alpha \beta} \quad$ the number of type $e$ containers in inventory at depot $d$ at the end of the period, given that the $\alpha^{\text {th }}$ realisation of supply, $\zeta_{d e}^{\alpha}$, and the $\beta^{\text {th }}$ realisation of demand, $\eta_{d e}^{\beta}$, holds.

$h_{d e}^{\alpha \beta} \quad$ the number of type $e$ containers short at depot $d$ at the end of the period, given that the $\alpha^{\text {th }}$ realisation of supply, $\zeta_{d e}^{\alpha}$, and the $\beta^{\text {th }}$ realisation of demand, $\eta_{d e}^{\beta}$, holds.

The stochastic objective of (1) is subsequently converted into its dual decomposition structure and expressed in (2).

$$
\min z=\sum_{s, d \in \mathbf{D}} \sum_{l \in \mathbf{L}} c_{s d l} n_{s d l}+\sum_{d \in \mathbf{D}} \sum_{e \in \mathbf{E}} \sum_{\alpha=1}^{R} \sum_{\beta=1}^{R}\left(p_{d e}^{\alpha} p_{d e}^{\beta}\right)\left(o_{d e} u_{d e}^{\alpha \beta}+r_{d e} h_{d e}^{\alpha \beta}\right)
$$

The remainder of the formulation is also provided in its dual decomposition structure. Each container relocated must be assigned to a specific truck type, hence the introduction of (3).

$$
x_{\text {sde }}=\sum_{l \in \mathbf{L}} y_{\text {sdel }} \quad \forall s, d \in \mathbf{D}, e \in \mathbf{E}
$$

To ensure that we do not exceed either the weight or volumetric capacity of any truck, we introduce (4) and (5) respectively.

$$
\begin{array}{ll}
\sum_{e \in \mathbf{E}} w_{e}^{C} y_{s d e l} \leq W_{l}^{T} n_{s d l} & \forall s, d \in \mathbf{D}, l \in \mathbf{L} \\
\sum_{e \in \mathbf{E}} v_{e}^{C} y_{s d e l} \leq V_{l}^{T} n_{s d l} & \forall s, d \in \mathbf{D}, l \in \mathbf{L}
\end{array}
$$

Assuming that relocations are affected concurrently with supply and demand realisations, and managed operationally in an efficient manner, we introduce the inventory and shortage calculation constraint of (6).

$$
\begin{aligned}
& u_{d e}^{\alpha \beta}-h_{d e}^{\alpha \beta}=I_{d e}+\sum_{s \in \mathbf{D}} x_{s a e}-\sum_{d \in \mathbf{D}} x_{a d e}+\zeta_{a e}^{\alpha}-\eta_{a e}^{\beta} \forall a \in \mathbf{D}, e \in \mathbf{E} \\
& \forall \alpha, \beta \in\{1, \ldots, R\}
\end{aligned}
$$


The volumetric capacity of the depots is also considered. With (7) we employ a somewhat robust approach, and ensure that volumetric capacity is always adhered to, even in highsupply-low-demand scenarios.

$\sum_{e \in \mathbf{E}} v_{e}^{C} u_{d e}^{\alpha \beta} \leq V_{d}^{D} \quad \forall d \in \mathbf{D}, \alpha, \beta \in\{1, \ldots, R\}$

Lastly, we impose nonnegativity and integrality constraints in (8) through (11).

$$
\begin{array}{ll}
u_{d e}^{\alpha \beta}, h_{d e}^{\alpha \beta} \geq 0 & \forall d \in \mathbf{D}, e \in \mathbf{E}, \alpha, \beta \in\{1, \ldots, R\} \\
x_{s d e} \geq 0 & \forall s, d \in \mathbf{D}, e \in \mathbf{E} \\
y_{s d e l} \geq 0 & \forall s, d \in \mathbf{D}, e \in \mathbf{E}, l \in \mathbf{L} \\
n_{s d l} \geq 0 \text { and integer } & \forall s, d \in \mathbf{D}, l \in \mathbf{L}
\end{array}
$$

\section{NUMERICAL EXAMPLE}

This section provide a numerical example based on the 6-depot situation for a South African container provider. The example considers the network with $D=6$ depots and $L=6$ truck types. In Section 1 it was noted that 'container' is used in this document as the collective name for a wide variety of different reusable packaging types, and not only for actual containers; therefore the number of different container types in this example was set to $E=48$. Parameter values have been simplified for the purpose of the example, and are the same for all container types. For example, the random supply at depot $d=1$ for all container types, denoted by $\tilde{\alpha}_{1 e}$, is approximated by a normal distribution with mean $\mu=2000$ and standard deviation $\sigma=50$; using the notation $\widetilde{\alpha}_{1 e}: N(2000,50) \quad \forall e \in \mathbf{E}$. Table 1 indicates the random supply and demand approximations for all depots.

\begin{tabular}{ccc}
\hline $\begin{array}{c}\text { Depot } \\
d\end{array}$ & Supply & $\begin{array}{c}\text { Demand } \\
\widetilde{\zeta}_{d e}\end{array}$ \\
\hline 1 & $N(2000,100)$ & $N(50,5)$ \\
2 & $N(500,50)$ & $N(500,50)$ \\
3 & $N(500,50)$ & $N(500,50)$ \\
4 & $N(2000,100)$ & $N(2000,100)$ \\
5 & $N(50,5)$ & $N(2000,100)$ \\
6 & $N(500,50)$ & $N(500,50)$ \\
\hline \multirow{4}{*}{ Demand for all container types $e \in \mathbf{E}$ is assumed to be similar. }
\end{tabular}

Table 1: Probability distributions for uncertain supply and demand ${ }^{\dagger}$

Each distribution is discretized into $R=5$ equidistant partitions, or intervals, each representing a singe realisation of the random variable. The partitions were created by generating an arbitrary large number $I=1000$ instances of the random variables according to the underlying distribution; establishing the minimum and maximum values; and sorting the instances into the calculated intervals. The resulting probabilities are calculated according to (12)

$p_{r}=\frac{n_{r}}{I} \quad \forall r=\{1, \ldots, R\}$ 
where $n_{r}$ denotes the number of the random variable instances in inteval $r$. The outcome for each interval is merely calculated as the expected value of the interval: the value associated with the centre of the interval. The resulting probabilities and outcomes are provided in Table 2.

\begin{tabular}{ccccccccc}
\hline Realisation & \multicolumn{2}{c}{$\mathbf{N}(2000,100)$} & & \multicolumn{2}{c}{$\mathbf{N}(500,50)$} & & \multicolumn{2}{c}{$\mathbf{N}(50,5)$} \\
\cline { 2 - 3 } \cline { 8 - 9 } \cline { 8 - 9 }$r$ & $p_{d e}^{r}$ & $\xi_{d e}^{r}$ & & $p_{d e}^{r}$ & $\xi_{d e}^{r}$ & & $p_{d e}^{r}$ & $\xi_{d e}^{r}$ \\
\hline 1 & 0.015 & 1712 & & 0.019 & 359 & & 0.036 & 38 \\
2 & 0.193 & 1850 & & 0.207 & 429 & & 0.241 & 44 \\
3 & 0.488 & 1988 & & 0.522 & 498 & & 0.464 & 50 \\
4 & 0.277 & 2126 & & 0.221 & 567 & & 0.227 & 57 \\
5 & 0.027 & 2264 & & 0.031 & 636 & & 0.032 & 63 \\
\hline
\end{tabular}

Table 2: Discretized probablities and outcomes for random distributions

Actual container data, in terms of weight and volume, has been used for the 48 different container types in this example, and typical truck and depot capacities were obtained from a company in South Africa. Actual transport costs were used, while inventory holding costs were approximated using management inputs. Since it was difficult to quantify stock-out instances from historical data, and also to put a monetary value on shortage costs, it was assumed to be a factor of 10 of the inventory holding cost. However, this shortage will be addressed again later in the paper.

The problem, referred to as the stochastic model (SM), was solved by coding it in GAMS and solving the model using the CPLEX solver, version 9.0 from iLOG, for 3600 CPU seconds on a standard desktop computer, after which the algorithm was interrupted, and the incumbent solution was chosen. Although the solution itself may not be of interest to the reader, the reliability of the approach may well be.

\section{MODEL RELIABILITY}

To compare the reliability of our proposed model with that of a model assuming deterministic forecasted demand, the proposed model is solved for a second time, but with a single realisation of the random demand, namely the expected value, which is, in the case of the normal distribution, the mean. The probability of the mean is taken as 1 since it is the only realisation. The model is referred to as the expected value model (EVM).

In this paper we use the term reliability to indicate whether the first stage relocation plan, provided by the optimisation model, will hold within given criteria in realistically generated scenarios. Say we generate a scenario consisting of one instance for each random variable based on its probabilistic distribution. For the scenario, we calculate what the actual inventory and recourse costs would have been, had we implemented the optimisation solution in that scenario. Reliability is then defined as 1 if either the actual cost is less than the expected cost provided by the optimisation model, or no shortages were incurred. The two criteria are mutually exclusive, so we only define the reliability to be 0 if both criteria are violated. Inventory was not considered to deem the solution unreliable even though it increases the total cost.

Even though the model formulation was based on an actual case study, a numerical example with arbitrary values is used for illustrative purposes. To establish a reliability measure, we repeat the reliability testing for 100 independent scenarios where an independent scenario represents a unique realisation of the random demand, and determine the percentage of reliable scenarios. The reliability results for both the SM and the EVM models are shown in Figure 1. Both models overspent in all scenarios: the SM on average by just over $0.3 \%$, and the EVM by nearly $1.9 \%$. The main overspend, however, was on inventory holding cost, and not necessarily on shortage costs. This is confirmed when considering the reliability measure: $93 \%$ for the SM, and $74 \%$ for the EVM. 


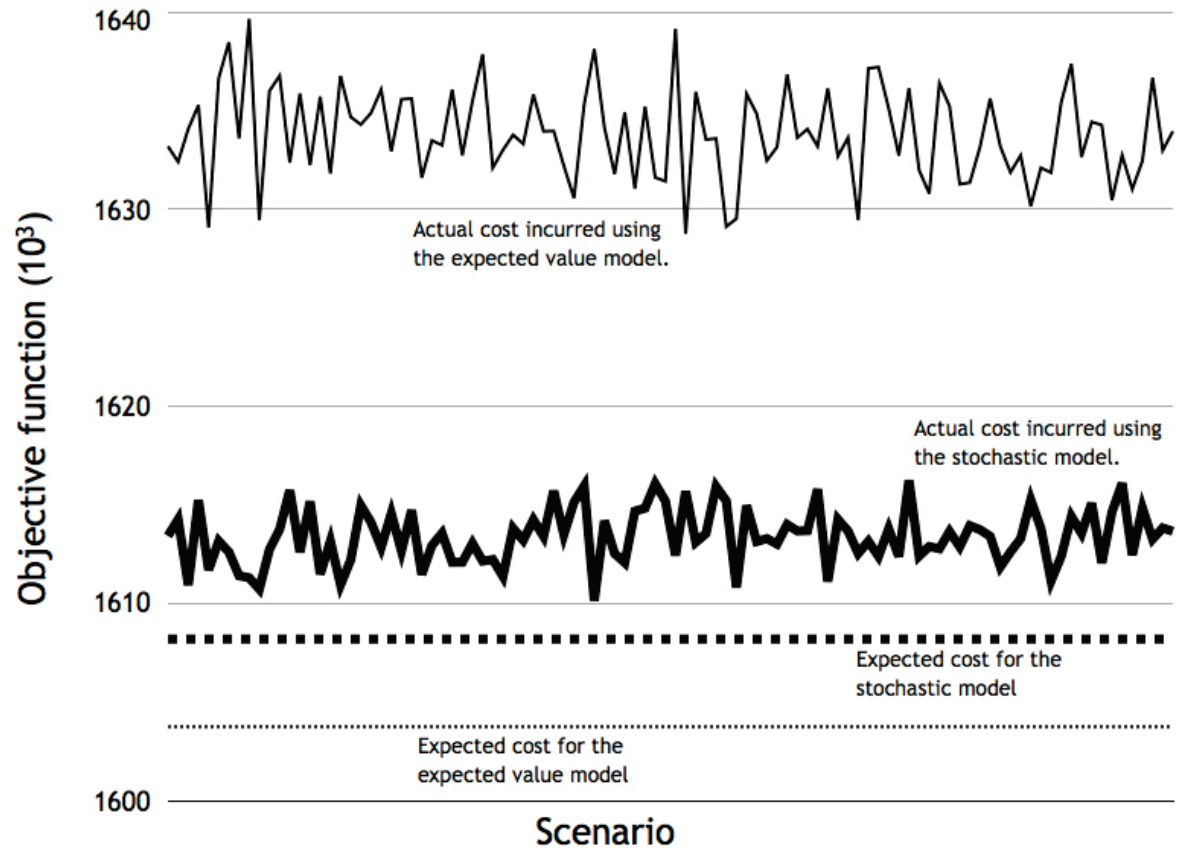

Figure 1: Reliability comparison of the proposed stochastic model versus the expected value model

It is expected that the objective function of the SM, i.e. the expected total cost, will be higher than the expected cost for the EVM, since the former makes decisions by taking multiple uncertain supply and demand realisations into account. Industry tends often to rely on such objective functions, and to price its products and/or services accordingly. The higher actual costs incurred by the EVM indicate not only that too much inventory is kept, but also that such inventory is kept at the wrong depots, resulting in shortages elsewhere in the network, which in turn incur recourse costs in the form of expedited deliveries.

But reliability figures of both the SM and EVM indeed rely on an informed choice of the weight, or unit cost, of the recourse components - namely, the inventory and shortage costs. To analyse the sensitivity, we performed multiple reliability tests, each time with a different ratio of shortage cost to inventory holding cost. The result of the sensitivity analysis is illustrated in Figure 2. The larger the difference between the two weights, the more reliable the SM. Even in the worst case, where the recourse weights are equal, the SM outperforms the EVM with a reliability of $87 \%$ to $77 \%$.

\section{CONCLUSION}

Reusable packaging may imply more environmentally friendly alternatives to packing, storing, and handling products, but its cost management requires careful consideration to ensure it does not impinge on profitability. The network for reusable packaging is typically unbalanced, requiring empty containers to be relocated throughout the network. Such relocations incur cost, but yield no revenue, emphasising the need for analytical cost minimisation decision support when planning relocations.

The state-of-practice in industry is often to forecast supply and demand in uncertain environments, and then to use the forecasted values as deterministic parameters in decision-making models. Such models are referred to as expected value models. In this paper a stochastic model is proposed as an alternative, and we show that such stochastic 
models outperform their expected value counterparts, even more so when there is a large differential between the inventory holding cost and shortage cost.

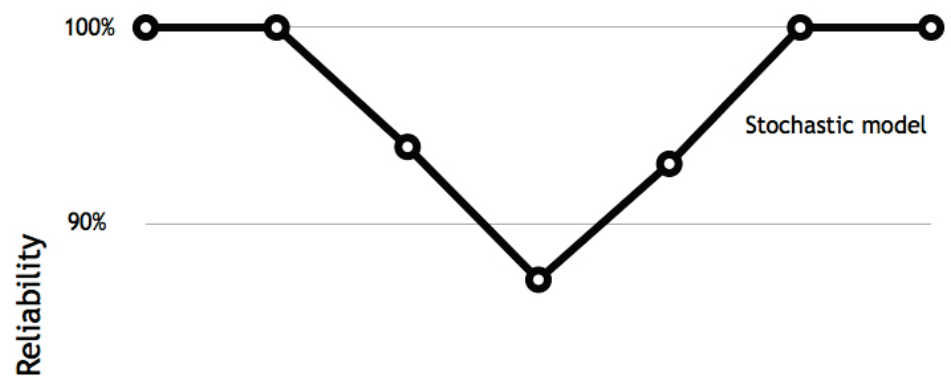

$80 \%$

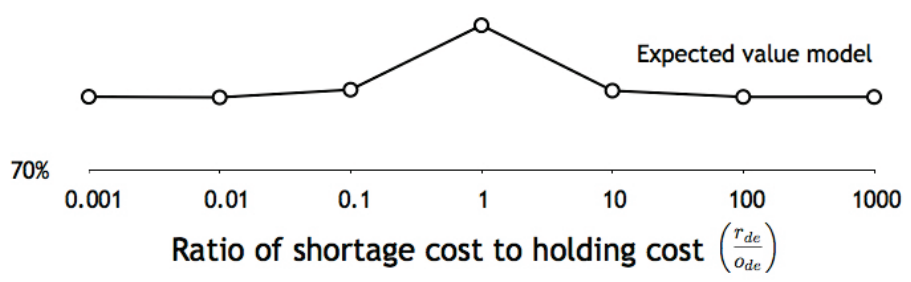

Figure 2: Sensitivity of the reliability measure for different shortage cost to inventory holding cost ratios

Not only does the stochastic model provide more reliable solutions, it also provides a better input on which companies can base their pricing for products and services. Merely inflating the inventory throughout the network to account for uncertainties (often referred to as fat solutions) typically results in containers being kept at the wrong depots even though more containers are in circulation. Since reusable containers are more costly than their consumable and/or recyclable counterparts, a larger number of containers in circulation implies tied up capital.

Stochastic models as proposed in our paper are by no means the Holy Grail of network optimisation, and three areas of further research are suggested. First, it is assumed that the uncertain supply and demand follows probabilistic distributions, and that such distributions are known with certainty. In the presence of sufficient historical data, as was the case for the industry partner on whose situation this paper was based, one could establish container-location-specific distributions. However, when intermittent or erratic demand occurs, or where sufficient historical data is absent, the reliabilities cannot necessarily be guaranteed. One suggestion would be to investigate the application of fuzzy programming. Another would be not to approximate random distributions, but rather to generate probability-outcome realisations influenced by expert and management opinion.

Second, the problem considered in this paper only accounts for a single time period. The formulation can be extended to take multiple time periods into account. The formulation of such problems can become somewhat messy, since each recourse variable becomes a function not only of the uncertainties of the current period, but also of all prior time periods. Birge [1] suggests a decomposition approach for such multistage stochastic linear programs. This brings us to the third suggestion for further research.

Computationally, complexity is adversely affected when more and more complex models are formulated. One must be careful that the time to find a solution to a problem instance is not longer than the useful life of the solution (if found at all). An alternative approach to coding the models mathematically in search of exact solutions, heuristic - and more specifically, metaheuristic - approaches should be investigated. With proper lower bounds, 
such approximate solutions may prove to result in very usable solutions to realistic problems in industry.

\section{REFERENCES}

[1] Birge, J.R. 1985. Decomposition and partitioning methods for multistage stochastic linear programs, Operations Research, 33(5), pp 989-1007.

[2] Birge, J.R. \& Ho, J.K. 1993. Optimal flows in stochastic dynamic networks with congestion, Operations Research, 41(1), pp 203-216.

[3] Cheung, R.K. \& Chen, C.Y. 1998. A two-stage stochastic network model and solution methods for the dynamic empty container allocation problem, Transportation Science, 32(2), pp 142-162.

[4] Cheung, R.K. \& Powell, W.B. 1996. An algorithm for multistage dynamic networks with random arc capacities, with an application to dynamic fleet management, Operations Research, 4(6), pp 951-963.

[5] Choong, S.T., Cole, M.H. \& Kutanoglu, E. 2002. Empty container management for intermodal transportation networks, Transportation Research Part E, 38(6), pp 423438.

[6] Coyle, J.J., Bardi, E.J. \& Langley, C.J. Jnr. 2003. The management of business logistics: A supply chain perspective, $7^{\text {th }}$ edition, Thomson Learning.

[7] Crainic, T.G., Gendreau, M. \& Dejax, P. 1993. Dynamic and stochastic models for the allocation of empty containers, Operations Research, 41(1), pp 102-126.

[8] Crainic, T. \& Delorme, L. 1993. Dual-ascent procedures for multicommodity location-allocation problems with balancing requirements, Transportation Science, 27(2), pp 90-101.

[9] Dejax, P.J. \& Crainic, T. 1987. A review of empty flows and fleet management models in freight transportation, Transportation Science, 21(4), pp 227-248.

[10] Du, Y. \& Hall, R. 1997. Fleet sizing and empty equipment redistibution for centerterminal transportation networks, Management Science, 43(2), pp 145-157.

[11] Hall, R. 1999. Stochastic freight flow patterns: Implications for fleet optimization, Transportation Research Part A, 33(6), pp 449-465.

[12] Holmberg, K., Joborn, M. \& Lundgren, J.T. 1998. Improved empty freight car distribution, Transportation Science, 32(2), pp 163-173.

[13] Misra, S. 1972. Linear programming of empty wagon disposition, Rail International, 3, pp 217-232.

[14] Olivo, A., Zudas, P., Francesco, M.D. \& Manca, A. 2005. An operational model for empty container management, Maritime Economics and Logistics, 7, pp 199-222.

[15] Rajgopal, J. 2004. Principles and applications of operations research, In Maynard's Industrial Engineering Handbook, McGraw-Hill. 